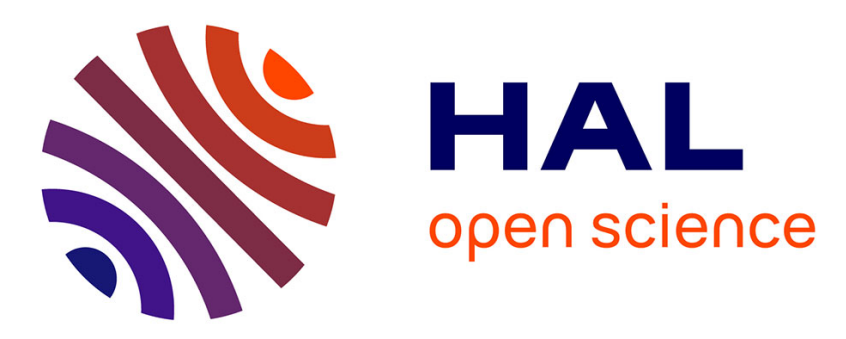

\title{
Autisme, psychose précoce, troubles envahissants du développement
}

\author{
C. Bursztejn, J.-P. Raynaud, R. Misés
}

\section{To cite this version:}

C. Bursztejn, J.-P. Raynaud, R. Misés. Autisme, psychose précoce, troubles envahissants du développement. Annales Médico-Psychologiques, Revue Psychiatrique, 2011, 10.1016/j.amp.2011.03.011 . hal-00748143

\section{HAL Id: hal-00748143 \\ https://hal.science/hal-00748143}

Submitted on 5 Nov 2012

HAL is a multi-disciplinary open access archive for the deposit and dissemination of scientific research documents, whether they are published or not. The documents may come from teaching and research institutions in France or abroad, or from public or private research centers.
L'archive ouverte pluridisciplinaire HAL, est destinée au dépôt et à la diffusion de documents scientifiques de niveau recherche, publiés ou non, émanant des établissements d'enseignement et de recherche français ou étrangers, des laboratoires publics ou privés. 


\section{Accepted Manuscript}

Title: Autisme, psychose précoce, troubles envahissants du développement

Authors: C. Bursztejn, J.-P. Raynaud, R. Misés

PII: $\quad$ S0003-4487(11)00076-X

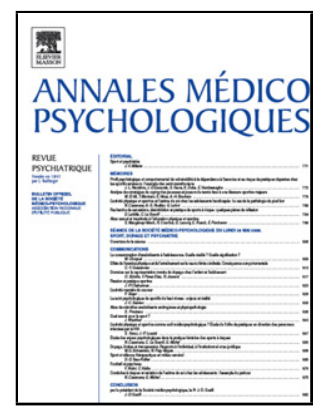

DOI: $\quad$ doi:10.1016/j.amp.2011.03.011

Reference: $\quad$ AMEPSY 1317

To appear in: $\quad$ Annales Médico-Psychologiques

Please cite this article as: Bursztejn C, Raynaud J-P, Misés R, Autisme, psychose précoce, troubles envahissants du développement, Annales medio-psychologiques (2008), doi:10.1016/j.amp.2011.03.011

This is a PDF file of an unedited manuscript that has been accepted for publication. As a service to our customers we are providing this early version of the manuscript. The manuscript will undergo copyediting, typesetting, and review of the resulting proof before it is published in its final form. Please note that during the production process errors may be discovered which could affect the content, and all legal disclaimers that apply to the journal pertain. 


\section{Communication}

Autisme, psychose précoce, troubles envahissants du développement Autism, early psychosis, pervasive developmental disorders

C. Bursztejn ${ }^{\text {a }}$, J.-P. Raynaud ${ }^{b}$, R. Misés ${ }^{c}$

a) CHRU Hôpital civil, 1 place de l'Hôpital, 67091 Strasbourg cedex, France

b) CHU de Toulouse Hôpital La Grave, TSA 60033, 31059 Toulouse cedex 9, France

c) 21 rue Barbet de Jouy, 75007 Paris, France

Auteur correspondant : Dr Jean-Philippe Raynaud, CHU de Toulouse Hôpital La Grave, TSA 60033, 31059 Toulouse cedex 9, France

Adresse email : raynaud.jp@chu-toulouse.fr

\section{Résumé}

Dans la nouvelle version de la classification française des troubles mentaux de l'enfant et de l'adolescent (CFTMEA-R2010), la notion de psychose précoce a été conservée dans le cadre de la catégorie "Troubles envahissants du développement (TED)», définis par les classifications internationales (DSM-IV et CIM-10). Au sein des TED, les catégories diagnostiques «Psychose précoce déficitaire» (« retard mental avec troubles autistiques ou psychotiques») et «Dysharmonie Multiple et Complexe du Développement (MCDD) Dysharmonie psychotique », restent individualisées. Un effort particulier a été fait pour faciliter les correspondances entre ces catégories diagnostiques de la CFTMEA-R2010 et de la CIM-10, en vue du relevé informatisé d’activité (RIM-Psy) et des travaux de recherche internationaux.

Mots clés : Adolescent ; Autisme ; Classification ; Enfant ; Psychose

\section{Abstract}

In the new revision of the French classification of mental disorders in children and adolescents (CFTMEA-R2010), the concept of early psychosis has been preserved in the category "Pervasive Developmental Disorders" (PDD), as defined by the international classifications (DSM-IV and ICD-10). Within PDD, diagnostic categories "Early psychosis 
with retardation" (and "mental retardation with autistic or psychotic disorders") and "Multiple and Complex Development Disharmony (MCDD) - Psychotic Disharmony” are still individualized. A special effort was made to facilitate connections of the diagnostic categories between CFTMEA-R2010 and ICD-10. These connections are useful for activity computerized recording of (RIM-Psy) and international research programs.

Keywords: Adolescent; Autism; Child; Classification; Psychosis

Le DSM-III, publié en 1981, a pratiquement éliminé de sa nomenclature le terme de psychose [22]. Pour désigner ce qui entrait dans le champ des psychoses spécifiques de l'enfant, un nouveau terme a été créé : pervasive developpemental disorders (PDD), d'abord traduit en français par «troubles globaux du développement», puis par «troubles envahissants du développement » (TED), à partir du DSM-III-R. Au-delà de ce changement de terminologie, c’est toute la conception de ces troubles précoces qui a été profondément modifiée : l'autisme, jusque-là considéré comme un syndrome rare à côté d'autres formes de psychoses précoces, devenait la forme centrale, générique, en quelque sorte le prototype des troubles graves et précoces du développement de l'enfant [2].

Cette évolution s'est encore accentuée avec le DSM-IV, dans lequel seul le trouble autistique a bénéficié d'une description et de critères [3]. Tout autre aspect des troubles envahissants du développement s’est trouvé rejeté dans la catégorie des «troubles non spécifiés », dont il était pourtant reconnu qu'elle correspond à un nombre de cas plus important que le trouble autistique lui-même [12].

La CIM-10, parue en 1993, a entériné la notion de troubles envahissants du développement et la place de premier plan accordée à l'autisme infantile [19,22]. Elle a cependant adopté une position assez différente en introduisant d'autres catégories dans ce chapitre :

- le syndrome d’Asperger, pour désigner les troubles autistiques sans retard mental important ;

- le syndrome de Rett, répondant à une encéphalopathie évolutive dont l'origine génétique a été mise en évidence ;

- les autres troubles désintégratifs ; 
- les troubles hyperactifs avec retard mental et stéréotypies, dont la validité clinique était mise en doute par les rédacteurs de la CIM eux-mêmes et qui, depuis, n’ont quasiment pas fait l'objet d'études ou de publications.

Le DSM-IV s'est pratiquement aligné sur la présentation de la CIM-10, à l'exception de cette dernière sous-catégorie, probablement vouée à disparaître [10].

Par rapport à ces changements, reflets des débats et controverses sur l'autisme et sur ce qu'il est convenu d'appeler les « troubles associés », la CFTMEA présente un tableau assez radicalement différent [13,14]. À côté de l'autisme infantile, dont elle marque l'importance et la spécificité, elle décrit d'autres formes de psychoses précoces, à la suite des recherches en psychopathologie, particulièrement développées sur ce thème dans notre pays [20].

La notion de " psychose précoce déficitaire » permet d'aborder, de manière spécifique, le problème de l'intrication, fréquente, d'un retard mental avec des manifestations et des mécanismes autistiques ou psychotiques.

Les dysharmonies psychotiques, individualisées par Roger Misès, correspondent à des formes dans lesquelles l'analyse psychopathologique révèle des éléments psychotiques sous une symptomatologie incluant des retards et des dysharmonies des acquisitions psychomotrices et cognitives, associées à des troubles du comportement.

\section{1. Évolution de la CFTMEA dans le champ de l’autisme et des psychoses précoces}

(tableau 1)

Dans ce contexte, les similitudes d'ordre clinique entre l'autisme et les psychoses précoces ont été retenues dans la CFTMEA, avec dans ce cadre, les TED pour lesquels l’équivalence était possible [5,15,16].

On retrouve ainsi la distinction entre l'autisme infantile précoce type Kanner et les autres formes de l'autisme. Les psychoses précoces déficitaires, avec une correspondance pour le retard mental avec troubles autistiques ou psychotiques. Les dysharmonies psychotiques, sur lesquelles nous reviendrons [14].

Deux nouvelles catégories ont été introduites :

- le syndrome d'Asperger : ce syndrome, dont l'autonomie par rapport à l'autisme et les limites précises sont encore discutées, fait l'objet de nombreuses recherches et publications ; 
- les troubles désintégratifs de l'enfance, dont le syndrome de Rett n’apparaît être aujourd'hui qu'un cas particulier.

En définitive, on retrouve dans la CIM-10 et la CFTMEA-R2010 des dénominations identiques ou très voisines (voir tableau 2), ce qui a facilité la mise en place d'un tableau de correspondance pour le relevé informatisé de l’activité médicale (RIM-Psy) [1].

Restent cependant les dysharmonies psychotiques de la CFTMEA, qui n’ont pas de correspondance dans la CIM-10. Les enfants concernés se trouvent classés soit «autres TED » dans la CIM-10, soit « TEDNS » dans le DSM-IV. Cela soulève de sérieux problèmes car toutes les études épidémiologiques montrent la place importante - environ $30 \%$ - tenue dans le groupe «Autisme, psychoses précoces » par les dysharmonies psychotiques qui possèdent, en outre, d'indéniables particularités cliniques et appellent de ce fait des interventions adaptées, susceptibles de mener à une réintégration complète.

Il faut rappeler brièvement l'importance et les éléments distinctifs des dysharmonies psychotiques. Comme Jacques Hochmann le rappelait récemment: «Ce diagnostic n’implique aucune prise de position étiologique univoque [11]. Il envisage de manière épigénétique une interaction entre les facteurs d'environnement et les facteurs neurobiologiques dans un processus évolutif. La symptomatologie est polymorphe, associant ou alternant des éléments de retrait et d'inhibition avec des éléments d'excitation et d'instabilité. L'enfant est surtout étrange et ses capacités cognitives et adaptatives en damier peuvent être de bon niveau dans certains secteurs particulièrement investis et médiocres dans d'autres. Elles contrastent avec le maintien de certaines fixations archaïques sur l'usage de la bouche, du tact ou de l'odorat. Surtout, à travers des symptômes polymorphes, l'enfant manifeste des angoisses massives qui peuvent prendre l'aspect de phobies localisées ou généralisées vis-à-vis du monde extérieur ou d'inquiétudes portant sur l'intégrité corporelle, parfois contrôlées par des mécanismes de type obsessionnel. Ces angoisses sont favorisées par une infiltration de la réalité par un imaginaire souvent cru qui, projeté sur les objets environnants, maintient l'immersion dans un univers peu rassurant sinon terrifiant. »

Les orientations de la CIM-10 n’ont pas seulement assuré l'élimination des dysharmonies psychotiques, elles ont conduit, plus largement, à faire entrer dans les «Troubles du spectre de l'autisme » ou dans le cadre élargi des TED, de très nombreux enfants qui selon nous devraient être classés dans d'autres catégories nosographiques : les dépressions précoces, les troubles sévères dans l'organisation des fonctions cognitives ou du langage, les pathologies du développement survenant chez le très jeune enfant et qui 
comportent parfois un risque d'entrer dans l'autisme mais sans que cela constitue d'emblée une fatalité.

Par ailleurs, il faut souligner, comme l'a fait dès 1997 Sylvie Tordjman, la convergence entre dysharmonie psychotique et un concept introduit dès 1986 par le Yale Child Study Center: le Multiple Complex Developmental Disorder ou MCDD [6,21]. On notera que l'objectif de l'équipe de Yale était initialement d'éviter le recours abusif au diagnostic de Trouble déficitaire de l'attention avec hyperactivité (TDAH) qui commençait à être très en présent outre-Atlantique. Les critères proposés pour ce diagnostic de MCDD incluent des troubles de la régulation des affects et de l'anxiété, des troubles du comportement social, une altération des processus cognitifs à type de troubles de la pensée, une apparition des symptômes dans les premières années de vie, et excluent un processus autistique ou schizophrénique. À ce jour, cette catégorie diagnostique, qui offre d’indéniables similitudes avec celle des «Dysharmonies psychotiques » de la CFTMEA, n’a été introduite ni dans le DSM ni dans la CIM-10. Récemment, à l’occasion des échanges en vue de l'élaboration du DSM-V, la Société Française de Psychiatrie de l'Enfant et de l'Adolescent a adressé à l'Association américaine de psychiatrie un commentaire argumenté visant à soutenir l'introduction des Multiplex Developmental Disorder (MDD) dans la nouvelle version de la classification américaine.

\section{Perspectives}

Ce que nous observons aujourd'hui, si nous nous référons au Recueil d'Informations Médicalisées en Psychiatrie, ou aux registres du handicap, qui sont renseignés sur la base de la CIM-10, c’est une «épidémie » de Troubles envahissants du développement, identique à celle que Fombonne décrit depuis des années tout autour de la planète $[4,9,17,18]$ et qui semble clairement progresser au même rythme que l'extension des critères de diagnostic. Sur le plan épidémiologique, le processus est en marche. L’introduction probable dans le DSM-V et dans la CIM-11 de la catégorie "Troubles du spectre autistique », en lieu et place de la catégorie "Troubles envahissants du développement » va accentuer encore le phénomène de globalisation syndromique. Nous craignons également que, confrontés à la question du « chaînon manquant » dans les classifications, les cliniciens n'aient recours à d'autres catégories diagnostiques comme la schizophrénie, et que certaines catégories ne se trouvent, à terme, artificiellement modifiées, ou que l'évolution à l'adolescence et à l'âge adulte d'un certain nombre de troubles dont la dimension psychotique n’aurait pas été suffisamment prise 
en compte ne soit encore plus pénalisante pour des enfants et des familles. N’assiste-t-on pas d'ores et déjà à des formes d'exclusion catastrophiques de certaines institutions qui n'ont pas pu prendre la mesure de cette dimension et de sa part dans l'expressivité et l'évolutivité des symptômes les plus bruyants ? C’est bien là le plus important : sur le plan clinique, sur le terrain, au niveau de chaque enfant, chaque adolescent, chaque diagnostic, chaque projet individualisé, nous défendons l'intérêt d'une approche multidimensionnelle et coordonnée, régulièrement réajustée, harmonisée et explicitée aux parents, à la personne concernée [7]. Cette approche multidimensionnelle nous semble à privilégier autant sur le plan de l'évaluation, qui a pris une grande importance avec la création des Centres Ressources Autisme, que sur le plan des soins, des accompagnements et des projets proposés aux personnes avec TED et à leurs familles [8]. Il ne s’agit pas ici de défendre une idéologie ou une approche théorique, mais une certaine idée de la complexité de l'être humain, de la nuance et de la complémentarité des approches exploratoires et thérapeutiques. La version 2010 de la CFTMEA propose aux cliniciens davantage de critères différentiels, notamment pour les enfants dont les troubles ne se situent ni dans le cadre des TED classiquement décrits ni dans ses frontières. Elle nous semble, dans cette optique, à même de répondre au souci de la communauté pédopsychiatrique de prendre en compte dans le diagnostic et les soins la perspective catégorielle et dimensionnelle tout en faisant le lien avec la CIM-10 [8].

\section{Conflit d'intérêt : à compléter par l'auteur}

\section{Références}

[1] Agence technique de l'information sur l'hospitalisation (ATIH). Mise à disposition des outils de codage des diagnostics en psychiatrie. www.atih.sante.fr , 19 février 2007.

[2] Allen DA, Steinberg M, Dunn M, Fein D, Feinstein C, Waterhouse L, Rapin I. Autistic disorders versus other pervasive developmental disorders in young children: same or different? Eur Child Adolesc Psychiatry 2001;10:67-78.

[3] American Psychiatric Association. DSM-IV, Manuel Diagnostique et Statistique des Troubles Mentaux. Paris: Masson; 1996.

[4] Autism and developmental disabilities monitoring network surveillance year 2002 principal investigators. Prevalence of autism spectrum disorders and developmental disabilities monitoring network, 14 sites, United States, 2002. MMWR Surveill Summ 2007;56:12-28. 
[5] Bursztejn C, Jeammet P. Autisme et psychoses de l'enfant dans la CFTMEA R-2000. Ann Méd Psychol 2002;160:216-9.

[6] Cohen DJ, Paul R, Volkmar FR. Issues in the classification of Pervasive and Other Developmental Disorders: toward DSMIV. J Am Aca Child Psychiatry 1986;25:213-20.

[7] Dupuis G. Classification de l'autisme et des psychoses précoces : point de vue du pédopsychiatre praticien en Centre Ressources Autisme. L’Information Psychiatrique 2010;86:215-21.

[8] Fédération française de psychiatrie et Haute Autorité de Santé. Recommandations pour la pratique professionnelle du diagnostic de l'autisme. www.has-sante.fr. Juin 2005.

[9] Fombonne E. Epidemiology of autistic disorders and other pervasive developmental disorders. J Clin Psychiatry 200;66:3-8.

[10] Harrington R. Diagnostic classification systems. Psychiatry 200;4:10-2.

[11] Hochmann J. Histoire et actualité du concept de psychose de l'enfant. L'Information Psychiatrique 2010;86:227-35.

[12] Mahoney WJ, Szatmari P, Maclean JE, Bryson SE, Bartolucci G, Walter SD, Jones MB, Zwaigenbaum L. Reliability and accuracy of differentiating pervasive developmental disorders subtypes. J Am Acad Child Adolesc Psychiatry 199;37:278-85.

[13] Misés R, et al. Classification française des troubles mentaux de l'enfant et de l'adolescent. Psychiatrie de l’enfant 1988;31:67-134.

[14] Misés R. Les dysharmonies psychotiques: une approche nosographique. Neuropsychiatrie de l'enfance et de l'adolescence 2000;48:396-401.

[15] Misés R, Quemada N. Classification française des troubles mentaux de l'enfant et de l'adolescent-R-2000. Classification internationale des maladies CIM10, Chapitre V, troubles mentaux et du comportement. CTNERHI, 2002 ( $4^{\mathrm{e}}$ éd.).

[16] Misés R, Garret-Gloanec N, Coinçon Y. Classification de l'autisme et des psychoses précoces, plaidoyer pour des convergences. L’Information Psychiatrique 2010;85:223-6.

[17] Moller LR, Sorensen MJ, Thompsen PH. ICD-10 classification in Danish child and adolescent psychiatry: have diagnoses changed after the introduction of ICD-10? Nord J Psychiatry 2007;61:71-8.

[18] Mussigbrodt H, Michels R, Malchow CP, Dilling H, Munk-Jorgensern P, Bertelsen A. Use of the ICD-10 classification in psychiatry: an international survey. Psychopathology 2000;33:94-9.

[19] Organisation Mondiale de la Santé. Classification CIM10 des troubles mentaux et de l'enfant et de l’adolescent. Traduction coordonnée par Marcelli D. Paris: Masson; 2001. 
[20] Quemada N. L'utilisation de la classification française des troubles mentaux de l'enfant et de l'adolescent dans une enquête nationale. Neuropsychiatrie de l'enfance et de l'adolescence 1990;38;540-5.

[21] Tordjman S, Ferrari P, Golse B, et al. Dysharmonies psychotiques et Multiplex Developmental Disorders, histoire d'une convergence. Psychiatrie de l'enfant 1997;40:473504.

[22] Volkmar FR, Cichetti DV, Bregman J, Cohen DJ. Three diagnostic systems for autism: DSM-III, DSM-III-R and ICD-10. J Autism Dev Disord 199;22:483-92.

Tableau 1 : CFTMEA-R2010 / CIM-10

\begin{tabular}{|c|c|c|c|}
\hline $\begin{array}{l}\text { CFTMEA- } \\
\text { R2010 }\end{array}$ & & $\begin{array}{l}\text { CIM- } \\
10\end{array}$ & \\
\hline 1.0 & $\begin{array}{l}\text { PSYCHOSES PRECOCES }- \text { TROUBLES } \\
\text { ENVAHISSANTS DU DEVELOPPEMENT }\end{array}$ & F84 & TED \\
\hline 1.00 & Autisme infantile précoce type Kanner & F84.0 & Autisme infantile \\
\hline 1.01 & Autres formes de l'autisme & F84.1 & Autisme atypique \\
\hline 1.02 & $\begin{array}{l}\text { Psychose précoce déficitaire, retard mental avec } \\
\text { troubles autistiques ou psychotiques }\end{array}$ & $\begin{array}{l}\text { F84.1 } \\
(+F 70- \\
79)\end{array}$ & $\begin{array}{l}\text { Autisme atypique } \\
+ \text { Retard mental }\end{array}$ \\
\hline 1.03 & Syndrome d'Asperger & F84.5 & $\begin{array}{l}\text { Syndrome } \\
\text { d'Asperger }\end{array}$ \\
\hline 1.04 & $\begin{array}{l}\text { Dysharmonie } \quad \text { Multiple et } \text { Complexe du } \\
\text { développement } \quad \text { (MCDD) }- \text { Dysharmonie } \\
\text { psychotique }\end{array}$ & F84.8 & Autres TED \\
\hline 1.05 & Troubles désintégratifs de l’enfance & F84.3 & $\begin{array}{l}\text { Autres troubles } \\
\text { désintégratifs de } \\
\text { l'enfance }\end{array}$ \\
\hline 1.08 & Autres psychoses précoces ou autres TED & F84.8 & Autres TED \\
\hline 1.09 & Psychoses précoces ou TED non spécifiés & F84.9 & $\begin{array}{l}\text { TED sans } \\
\text { précision }\end{array}$ \\
\hline
\end{tabular}


Tableau 2 : Evolution de la CFTMEA

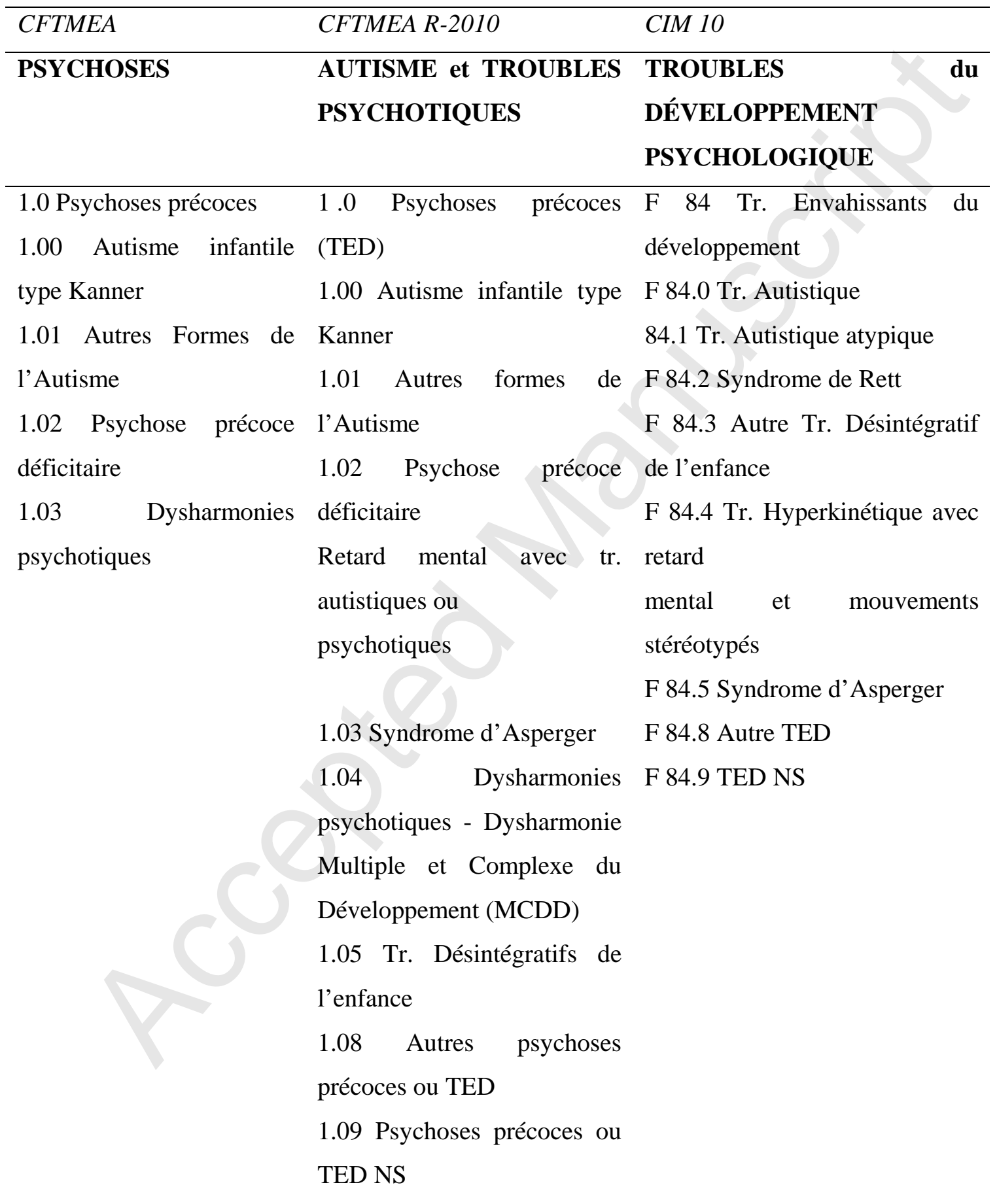

\section{Discussion}

